\title{
Regulation of Osteoblastic Differentiation: A Concept Note
}

\author{
Poonam Kanta ${ }^{1}$, Dheeraj Dumir ${ }^{2}$, Alka Khera ${ }^{3,}$ * \\ ${ }^{1}$ Department of Virology, Postgraduate Institute of Medical Education and Research, Chandigarh, India \\ ${ }^{2}$ Department of Cardiology, Max Super speciality Hospital, Mohali, Punjab, India \\ ${ }^{3}$ Department of Ophthalmology, Postgraduate Institute of Medical Education and Research, Chandigarh, India \\ Email address: \\ chauhan.poonamk@gmail.com (P. Kanta),dheeraj.dumir@gmail.com (D. Dumir),kheraalka@yahoo.com (A. Khera) \\ ${ }^{*}$ Corresponding author
}

\section{To cite this article:}

Poonam Kanta, Dheeraj Dumir, Alka Khera. Regulation of Osteoblastic Differentiation: A Concept Note. American Journal of Life Sciences. Vol. 6, No. 1, 2018, pp. 22-28. doi: 10.11648/j.ajls.20180601.14

Received: March 7, 2018; Accepted: June 22, 2018; Published: June 26, 2018

\begin{abstract}
Bone is endocrine metabolic organ which maintains its integrity through the process of bone remodeling controlled by several local and systemic factors. Any perturbation in the bone remodeling leads to development of osteoporosis which is mostly age related but can be premature as well. Several therapies have come up to treat osteoporosis including bisphosphonates but due to side effects natural compounds are looked like resveratrol which has estrogenic property depending upon the type of tissue it is acting upon. Resveratrol has stimulatory effect on bone cells by modulating the process of differentiation through change in the expression levels of osteoblastic genes through estrogen receptors.
\end{abstract}

Keywords: Bone Remodeling, Osteoporosis, Phytoestrogen, Resveratrol

\section{Introduction}

Osteoporosis is a well-defined disorder where estrogen plays an important role in bone remodelling and estrogen deficiency is the cause of postmenopausal osteoporosis. Hormonal replacement therapy could alleviate the postmenopausal osteoporosis but it has other undesirable side effects. Alternative therapy with naturally occurring phytoestrogen like resveratrol has been shown to have osteoprotective effects in animal model. This concise review focuses on the molecules which regulate bone remodelling directly or indirectly to maintain bone homeostasis.

\section{Bone Remodeling}

Bone remodelling is a lifelong process where old bone is replaced from the skeleton (bone resorption) by new bone (bone formation). This process controls the reshaping or replacement of bone during growth and injuries like fractures and also micro-damage, which occurs during normal activity. Bone building is stimulated by the secretion of growth hormone by the pituitary, thyroid hormone and sex hormones (estrogens and androgens) [1]. There are two types of cells which play role in bone remodelling, the osteoblastic cells (bone forming) and the osteoclastic cells (bone depleting). Osteoblasts are promoted to release various factors that indorse resorption of bone by stimulating osteoclast activity and its differentiation from progenitor cells [2].

According to National Osteoporosis Guideline Group (NOGG), osteoporosis is defined as loos of bone structure with weak strength due to deterioration of mineral content which increases the risk for fractures (https://www.sheffield.ac.uk/NOGG/NOGG\%20Guideline\% 202017.pdf). Osteoporosis is a major health problem affecting 2 in 3 women in postmenopausal years. A rapid decline in estrogen production is the leading cause of decrease in bone density immediately after menopause. Bone density is measured by means of many techniques, but the most common used one is Dual Energy X-ray absorptiometry (DEXA). The total bone mineral density (BMD) decreases by $30-40 \%$ at the age of 75 and this decrease is due to increase in osteoclastic activity which affect the bone quality. In clinics, this pathogenesis is detected only when it is symptomatic and it is difficult to replace this condition. 


\section{Pharmacological and Non-Pharmacological Interventions}

Pharmacological and non-pharmacological interventions are used to prevent or treat osteoporosis. Non-pharmacological interventions include a) change in life style b) increase in uptake of calcium, vitamin D and protein rich diet. Traditional therapy i.e. hormone replacement therapy (HRT) has been the first choice to prevent the bone loss but it is currently discouraged due to its side effects. As bone loss is highest soon after menopause, the traditional practice was to start the HRT before development of severe form of osteoporosis. For that the total concentration of estrogen is increased by giving combination of balanced estrogen with progestin. HRT treatment has been able to avert several deaths from 2002 to 2011 [3]. Other modalities used to treat post-menopausal osteoporosis have emphasized the use of anti-resorption agents such as estrogen, calcitonin and bisphosphonates.

\section{Estrogen and Bone Remodeling}

Bone is an estrogen sensitive tissue and change in the levels of estrogen affects the bone quality. Estrogen receptors have been described in all three different types of cells of bone (osteoblasts, osteocytes, and osteoclasts). It decreases rate of remodelling by decreasing osteoclast resorption and thus decreases bone formation rate [3]. Estrogen deficiency during post-menopausal years induces bone loss resulting in osteoporosis. Estrogen exerts its effects by either ER- $\alpha$ or ER- $\beta$. ER can function through either genomic DNA or nongenomic pathway where the need of binding to DNA is not required [4]. Alternatively, estrogen modulates bone formation and resorption markers through local or systemic factors. Disturbance in the rate of formation and resorption lead to loss of bone associated with osteoporosis and further restraining effect on osteoclastogenesis and osteoblastogenesis. ER (presumably ER- $\alpha$ ) was reported to be present on rat and human osteoblastic cells for the first time in 1988 [5].

Estrogen deficient condition in postmenopausal women is related to development of increased fracture risk and osteoporosis. To prevent osteoporosis estrogen is given in combination with Parathyroid hormone (PTH) as it is considered as reasonable approach. PTH increases the bone formation if used once. However, prolonged use of PTH inhibits the bone formation whereas alone estrogen inhibit the bone resorption by inhibiting osteoclastic activity [6]. Such drugs are also not useful to treat osteoporosis as they have many side effects not only in bone but other tissue also.

Combination therapy (estrogen+ bisphosphonate) has more promising effects on BMD than alone. Treatment of postmenopausal osteoporosis by HRT has many side effects like increased risk of developing breast cancer, cardiovascular disease and dementia. Thus, replacement of HRT with Selective Estrogen Receptor Modulators (SERM) or SERM like bazodoxifene needs to be considered. These compounds act as estrogen receptor agonist or antagonist depending upon type of tissue. Bazedoxifene (BZA) is a third generation SERM, developed from raloxifene but it is structurally different from raloxifene. BZA interacts with estrogen receptors in various tissues. However, this compound has also been shown to have many side effects [7].

\section{Phytoestrogens and Bone Metabolism}

Phytoestrogens are described as naturally occurring plant derived non-steroidal compounds that shows structural and functional similarity to steroidal estrogens. These phytoestrogens can act as agonist or antagonist depending on the type of tissue [8]. Many studies have demonstrated health benefits of phytoestrogens in their anticarcinogenic, neuroprotective, cardioprotective activities and bone formation promoting properties. Investigations on soy isoflavones (daidzein and genistein) were shown to have a beneficial effect on proliferation and differentiation of osteoblastic MC3T3-E1 cells [9]. Studies on avian osteoclasts showed that genistein inhibits bone resorption via tyrosine kinase inhibition, which regulate osteoclastic activity [10]. In earlier studies ovariectomized (OVX) animals were fed soy protein to study the effects of soy isoflavones on the bone loss and it was found that treatment with it could prevent bone loss [11] and this positive effect was later found to be due to the isoflavones in the soy [12]. Long term consumption of soy isoflavones also resulted in reversal of established bone loss [13]. Genistein and daidzein received attention as osteoprotective compounds and can be used for treatment of osteoporosis. They stimulate osteoblastic proliferation and differentiation and induce osteoclastic apoptosis by acting as inhibitor of tyrosin kinase. Genistein was shown to be effective in reducing bone loss in OVX rats. Genistein was also shown to have estrogenic effects on bone and bone marrow without exhibiting estrogenic action on the uterus [13]. Phytoestrogens also have many other beneficial effects like cardio-protective, neuro-protective and anticancer property apart from their positive effects on bone [14].

\section{Resveratrol and Bone Remodeling}

Recently in last few years resveratrol has attracted considerable attention due to its low toxicity and unique chemical structure. Resveratrol (3, 4, 5 -trihydroxystilbene), is a phytoalexin present in grapes, mulberries and peanuts. It is isolated from roots of Polygonum capsidatum(ko-jo-kon) in Japan. Resveratrol in ROB cells (Rat calvarias Osteoblastlike) could stimulate osteocalcin gene expression similar to estradiol without disrupting endocrine function [15]. Apart from its anti-resorptive property, resveratrol can enhance osteogenesis and inhibits adipogenesis. Resveratrol promotes osteogenesis through an increased expression of RUNX2 protein (a main regulator of osteoblastic differentiation) via promoting formation of active complex of Sirt1 and FOXO3A [16, 17]. Resveratrol stimulates osteoblastic cell 
proliferation at $1 \mu \mathrm{M}$ concentration with increased ALP activity and calcium deposition on day 12 . In the same study authors have shown that the proliferation and differentiation is through MAPK pathway [18]. Resveratrol treatment increases the ALP activity, osteocalcin, at doses up to 12.5 $\mu \mathrm{M}$ but not with higher dose. Thus, the study showed that there was increase in osteoblastic activity with resveratrol treatment without increasing cellular number thus promoting osteogenesis at very low concentrations [19]. Resveratrol was first demonstrated to directly stimulate cell proliferation and differentiation of osteoblasts in in vitro studies using osteoblastic MC3T3-E1 cells [20]. Liu et al and other authors have demonstrated that resveratrol alleviate the decrease in the bone mineral density (BMD) and bone calcium content (BCC) in OVX rat model which shows the beneficial effect on bone preservation [21, 22]. In a review Bagchi et al., 2001 suggested that resveratrol supplementation might be an alternative to HRT for its cardioprotective and osteoprotective effects and other potential health benefits in post-menopausal women [23]. Administration of resveratrol in trauma from occlusion C57BL/6 mice significantly shown decrease in osteoclastic genes [24].

\section{Estrogen Modulatory Effects of Resveratrol}

Resveratrol is categorized in a group of phytoestrogen due to its structural similarity to diethylstilbestrol. Resveratrol has been shown to bind to the estrogen receptor (ER) and activate the transcription of genes in ER- $\alpha$ overexpressed MCF-7 cell [25]. In addition to this resveratrol modulate the levels of TGF-1 $\beta$, IGF1 and TGF-2 $\beta$ mRNA [26]. Resveratrol can bind to both ER- $\alpha$ and $\beta$ with different affinity, but with 7000-fold lower affinity than E2 [27]. The estrogen modulatory activity of resveratrol depends on the estrogen response element (ERE) sequence and ER subtype. It brings about higher transcriptional activity when bound to ER- $\alpha$ than $\beta$. Resveratrol has shown to mediate antiestrogenic effects in endometrial cancer cells by selective down regulation of ER- $\alpha$ but not ER- $\beta$ [28, 29]. Estrogenic and anti-estrogenic effect of resveratrol depends on various factors such as structural conformation of the protein to which it binds, what kind of protein it interacts with and cell and tissue specificity [30].

\section{Factors Involved in the Process of Osteoblast Differentiation}

\subsection{ALP (Alkaline Phosphatase)}

Bone specific alkaline phosphatase ALP (EC 3.1.3.1) is synthesized by osteoblasts to remove phosphate group form proteins, DNA, RNA, or alkaloids [31]. ALP is associated with membrane and studies have shown that it is associated with transport processes and mineralization in bone. As ALP is a as marker for osteoblastic activity it is assessed to study the differentiation of mesenchymal stem cell or precursor osteoblasts in in vitro culture studies and in in vivo conditions $[32,33]$. ALP gene is the first one to be expressed during differentiation of osteoblasts. The expression of ALP is regulated by RUNX2, osterix and wnt signaling pathway [34]. E2 stimulates osteoblast differentiation when added intermittent to the culture as there is significant increase in ALP activity as compared to when the cells were given continuous E2 treatment [35]. Estradiol alone can induce osteoblastic differentiation albeit at slower rate but when given along with PTH, BMP4 and BMP7 the effect was more pronounced as in by significant increased expression of ALP $[6,36,37]$. But E2 can stimulate osteoblastic differentiation of bone marrow cells derived from ER- $\alpha$ Knockout mice at the same rate as for cell from wild type. These cells showed increased ALP activity with other differentiating markers. So, in such cases E2 functional effect is independent of estrogen receptors [38]. Phytoestrogens have positive effect on ALP activity of osteoblast and promote differentiation. Human bone marrow stem cells showed comparable ALP activity when treated with resveratrol and E2 at10 nM concentration [18].

\subsection{Osteopontin}

Osteopontin is a non-collagenous protein secreted by mature osteoblasts. It helps in bone remodelling by mineralization and is known as late stage osteoblastic marker of differentiation (Vanackeret al., 1998). Osteoblasts like cells expressing ER such as U2OS-ER $\alpha$ or U2OS-ER $\beta$ show increase in expression of ALP, osteocalcin, osteopontin and many other osteoblastic genes when treated with E2 for 24 and 48 hours. The increase in osteopontin expression is significant after 48 hours and it is more in cells expressing ER- $\beta$ [39].

\subsection{Osteocalcin}

Osteocalcin is a gamma-carboxylated protein synthesized by mature osteoblasts and released into circulation where it has functional effects on many tissues [40]. Estradiol treatment of rat primary osteoblast causes increase in expression of Col1A1, osteonectin, osteocalcin, osterix, RUNX2 and also promotes mineralization [41]. Flavonoids like resveratrol increase the expression col1A1, osteocalcin, ALP and RUNX2. This effect of resveratrol may or may not be through ERs. Resveratrol activates RUNX2 gene expression which intern increase expression of osteoblastic differentiation markers [17].

\section{Transcription Factors}

\subsection{RUNX2 (Run-Related Transcription Factor)}

RUNX 2 binds to DNA through its Runt domain and regulates gene expression. This transcription factor has binding sites in various genes like osteocalcin, Col1A1, bone sialoprotein and osteopontin. As soon as there is a signal for osteoblastic differentiation, the expression of RUNX2 increases which further stimulates the expression of various 
genes like Col1A1 and osteopontin, BSP and osteocalcin. The process of differentiation of osteoblast starts with increase in expression of RUNX2, osteocalcin and PTHR. The primary immortalized precursor osteoblastic cell line hFOB1.19 differentiates at higher temperature $39^{\circ} \mathrm{C}$ and this differentiation is marked with increase in expression of such markers [42]. Authors have found that resveratrol can increase the expression of RUNX2. This increase in expression is through stabilized active complex of SIRT1 /FOXO3A [17]. Estrogen increases the expression of RUNX2 and osteocalcin in early stages of differentiation but inhibits its expression in later stages. Hence, estrogen has stage specific modification of RUNX2 activity [43].

\subsection{Osterix (SP7)}

Ostrix is the Zinc finger transcription factor expressed during differentiation of osteoblast. Studies have shown that in osteosarcoma cells the expression level is significantly reduced and this reduction is responsible for development of tumor. Once the gene expression is restored in such cells, it can reduce the tumour growth. This suggests that osterix has role in decreasing proliferation and promotes the cells towards differentiation [44]. RUNX2 transcription factor regulates the expression of osterix. Osterix regulates the gene expression of Col1A1 gene which play role in differentiation of osteoblastic cells. It maintains the bone mineral density by regulating bone formation. Same study has shown that treatment of mesenchymal stem cells with BMP6 increase osterix expression level while there is little effect on RUNX2 [45].

\subsection{Aryl Hydrocarbon Receptor (AhR)}

$\mathrm{AhR}$ is a nuclear receptor which belongs to basic-helixloop-helix transcription factors. AhR is a inactive cytosolic transcription factor bound to several chaperones and becomes active when chemicals such as 2,3, 7, 8-tetrachlorodibenzop-dioxin (TCDD) interact with these receptors. Upon binding, the chaperones dissociate from the AhR and allow it to move inside the nucleus where it leads to the change in the transcription of osteopontin, BMP, Wnt, RUNX2, osteocalcin, and alkaline phosphatase [46, 47]. Cigarette smoke, which contain TCDD, reduces the bone mineral density and increases risk of bone fracture due to bone loss [48]. Initial evidence for a role of resveratrol on bone metabolism came indirectly from studies on the effect of AhR ligand (TCDD) present in cigarette smoke and other sources of air pollution on osteoporosis [49]. Transcripts for important bone associated proteins such as collagen type I, osteopontin, bone sialoprotein and alkaline phosphatase were reduced by AhR ligand. This effect was found to be antagonized by resveratrol, an already known antagonist of AhR [50], thereby identifying resveratrol as a promising therapeutic agent for smoking related bone loss. 3Methylcholanthrene, a polycyclic aromatic hydrocarbon (PAH) and AhR agonist, activates $\mathrm{AhR}$ to interact with unliganded ER- $\alpha$ and this entire AhR/ER- $\alpha$ complex is recruited to estrogen responsive genes, suggesting cross talk between ER- $\alpha$ and AhR [51]. AhR also has role in mammary gland tumorigenesis [52].

\subsection{TGF-1及 (Transforming Growth Factor Beta Family)}

TGF-1 $\beta$ has long list of members includes TGF-1 $\beta$, Nodal, Activin, BMPs. The signalling pathway starts when ligand binds to heteromeric serine/threonine kinase receptors on membranes. The signal passes to a member of Smad family and finally to nucleus. Non-classical pathway of TGF-1 $\beta$ also induces osteogenic differentiation by stimulating RUNX2 expression which is the main regulator of differentiation. TGF-1 $\beta$ has power of inducing differentiation of induced pluripotent stem cells (iPSC) into osteoblastic cells and bone formation in vivo. Combination of TGF-1 $\beta$ and retinoic acid (RA) induce differentiation to osteoblast by increasing RUNX2, osterix, osteopontin, and osteocalcin expression levels. These iPSC cells after treatment with TGF-1 $\beta$ and RA introduced bone formation in SCID mice [53]. There are some contradictory studies in which authors have shown that increase in TGF-1 $\beta$ levels in serum due to systemic inflammatory disease cause bone loss as there is decrease in ALP, osteocalcin, BMP2, BMP7, BSP2, OPG and increase in RANKL, osteopontin expression levels. This effect is reversed by blocking TGF-1 $\beta$ pathway. Thus, TGF-1 $\beta$ shows dual effect on bone [54].

\subsection{BMP2 (Bone Morphogenetic Protein 2)}

BMP2, a member of TGF- $\beta$ family is expressed in various tissue including osteoblasts, where it stimulates osteoblasts differentiation similar to RUNX2 transcription factor. Increase in BMP-2 expression in mesenchymal stem cells and precursor osteoblasts cause increase in differentiation and play important role in bone formation. BMPs exert their effect by inducing mesenchymal stem cell differentiation into osteoblast precursors. Every BMP has its own function like BMP2 is osteogenic where as BMP7 has a role in bone formation and regeneration after injury. BMP2/7 heterodimer has significant effect on regeneration and differentiation than alone. ALP, Col1A1 and osteocalcin were highly induced in presence of BMP $2 / 7$ heterodimer than homodimer of each used at lower dose [55]. E2 treatment cause increase in BMP2 gene expression. The increase in expression of BMP2 has direct effect of estrogen as ER along with estrogen can bind to its binding site in the promoter site of BMP2 [56]. Wnt/lrp5 and BMP2 are most potent stimuli for bone formation. Signalling pathway of BMP2 is similar to TGF- $1 \beta$ as both belong to the same family. BMP2 increases RUNX2 expression level by activating Smad1/5/8 and finally regulates expression of many osteoblastic genes like osteocalcin, osteopontin, collagen type 1 [57]. In precursor osteoblasts it induces differentiation but in mature cells it induces apoptosis [58]. BMP2 is required for postnatal bone growth as well. Authors have shown that BMP2 is required for good quality of bone formation. Knockout of BMP2 causes small length of bone with poor quality of bone 
material which increase the risk of fracture in these mice [59].

\subsection{BMP7 (Bone Morphogenetic Protein-7)}

BMP7 is member of TGF-1 $\beta$ superfamily involved in proliferation and differentiation of bone cells. It has promising effect in improving the bone loss in ovariectomized ovine model [60]. Administration of BMP-7 alone or in combination with E2 increases the cell proliferation, ALP activity with enhanced RUNX2 protein expression, followed by mineralization. All these osteoblastic features are significantly increased when BMP-7 alone is given as compared to E2 alone and this effect was more pronounced when given in combination with E2 [37].

\section{Conclusion}

Estrogen is known to be an important systemic factor regulating bone remodelling process and the estrogen deficient condition is responsible for postmenopausal osteoporosis. However, it is not clear whether resveratrol directly acts on bone to change the local factors of bone remoulding or whether some indirect mechanism involving systemic factors are involved. Elucidation of molecular mechanisms of osteoprotective effects of resveratrol can help us to understand the pathogenesis of osteoporosis in detail with clinical application of identification of molecular targets or the design of drugs that can be used to treat osteoporosis.

\section{Acknowledgements}

We acknowledge Dr. Thungapathra M. (Professor, Department of Biochemistry, PGIMER, Chandigarh) for her guidance.

\section{References}

[1] Manolagas, S. C., C. A. O'Brien, and M. Almeida, "The role of estrogen and androgen receptors in bone health and disease." Nat Rev Endocrinol, 2013. 9(12): p. 699-712.

[2] Crockett, J. C., et al., "Bone remodelling at a glance." J Cell Sci, 2011. 124(Pt 7): p. 991-8.

[3] Almeida, M., et al., "Estrogen receptor-alpha signaling in osteoblast progenitors stimulates cortical bone accrual." J Clin Invest, 2013. 123(1): p. 394-404.

[4] Syed, F. A., et al., "Distinct effects of loss of classical estrogen receptor signaling versus complete deletion of estrogen receptor alpha on bone." Bone, 2011. 49(2): p. 208-16.

[5] Compston, J. E., "Sex steroids and bone." Physiol Rev, 2001. 81(1): p. 419-447.

[6] Nasu, M., et al., "Estrogen modulates osteoblast proliferation and function regulated by parathyroid hormone in osteoblastic saos-2 cells: Role of insulin-like growth factor (igf)-i and igf-binding protein-5." J Endocrinol, 2000. 167(2): p. 305-13.
[7] Rossini, M., et al., "Profile of bazedoxifene/conjugated estrogens for the treatment of estrogen deficiency symptoms and osteoporosis in women at risk of fracture." Drug Des Devel Ther, 2013. 7: p. 601-10.

[8] Lagari, V. S. and S. Levis, "Phytoestrogens in the prevention of postmenopausal bone loss." J Clin Densitom, 2013. 16(4): p. 445-9.

[9] Wardlaw, G. M., "Putting osteoporosis in perspective." J Am Diet Assoc, 1993. 93(9): p. 1000-6.

[10] Sugimoto, E. and M. Yamaguchi, "Stimulatory effect of daidzein in osteoblastic mc3t3-el cells." Biochem Pharmacol, 2000. 59(5): p. 471-5.

[11] Blair, H. C., et al., "Variable effects of tyrosine kinase inhibitors on avian osteoclastic activity and reduction of bone loss in ovariectomized rats." J Cell Biochem, 1996. 61(4): p. 629-37.

[12] Arjmandi, B. H., et al., "Dietary soybean protein prevents bone loss in an ovariectomized rat model of osteoporosis." J Nutr, 1996. 126(1): p. 161-7.

[13] Arjmandi, B. H., et al., "Role of soy protein with normal or reduced isoflavone content in reversing bone loss induced by ovarian hormone deficiency in rats." Am J Clin Nutr, 1998. 68(6 Suppl): p. 1358S-1363S.

[14] Pilsakova, L., I. Riecansky, and F. Jagla, "The physiological actions of isoflavone phytoestrogens." Physiol Res, 2010. 59(5): p. 651-64.

[15] Rucinski, M., et al., "Estradiol and resveratrol stimulating effect on osteocalcin, but not osteonectin and collagen-1alpha gene expression in primary culture of rat calvarial osteoblastlike cells." Int J Mol Med, 2006. 18(4): p. 565-70.

[16] Zainabadi, K., C. J. Liu, and L. Guarente, "Sirt1 is a positive regulator of the master osteoblast transcription factor, runx2." PLoS One, 2017. 12(5): p. e0178520.

[17] Tseng, P. C., et al., "Resveratrol promotes osteogenesis of human mesenchymal stem cells by upregulating runx 2 gene expression via the sirt1/foxo3a axis." J Bone Miner Res, 2011. 26(10): p. 2552-63.

[18] Dai, Z., et al., "Resveratrol enhances proliferation and osteoblastic differentiation in human mesenchymal stem cells via er-dependent erk1/2 activation." Phytomedicine, 2007. 14(12): p. 806-14.

[19] Erdman, C. P., et al., "Effects of resveratrol on enrichment of adipose-derived stem cells and their differentiation to osteoblasts in two-and three-dimensional cultures." J Tissue Eng Regen Med, 2012. 6 Suppl 3: p. s34-46.

[20] Mizutani, K., et al., "Resveratrol stimulates the proliferation and differentiation of osteoblastic mc3t3-el cells." Biochem Biophys Res Commun, 1998. 253(3): p. 859-63.

[21] Liu, Z. P., et al., "Effects of trans-resveratrol from polygonum cuspidatum on bone loss using the ovariectomized rat model." J Med Food, 2005. 8(1): p. 14-9.

[22] Xi, H., et al., "[effect of resveratrol on peak bone mass in growing rats]." Zhejiang Da Xue Xue Bao Yi Xue Ban, 2017. 46(6): p. $578-584$.

[23] Bagchi, D., et al., "Benefits of resveratrol in women's health." Drugs Exp Clin Res, 2001. 27(5-6): p. 233-48. 
[24] Matsuda, Y., et al., "Resveratrol suppresses the alveolar bone resorption induced by artificial trauma from occlusion in mice." Oral Dis, 2018. 24(3): p. 412-421.

[25] Gehm, B. D., et al., "Resveratrol, a polyphenolic compound found in grapes and wine, is an agonist for the estrogen receptor." Proc Natl Acad Sci U S A, 1997. 94(25): p. 1413843 .

[26] Basly, J. P., et al., "Estrogenic/antiestrogenic and scavenging properties of (e)- and (z)-resveratrol." Life Sci, 2000. 66(9): p. 769-77.

[27] Bowers, J. L., et al., "Resveratrol acts as a mixed agonist/antagonist for estrogen receptors alpha and beta." Endocrinology, 2000. 141(10): p. 3657-67.

[28] Bhat, K. P., et al., "Estrogenic and antiestrogenic properties of resveratrol in mammary tumor models." Cancer Res, 2001. 61(20): p. 7456-63.

[29] Bhat, K. P. and J. M. Pezzuto, "Resveratrol exhibits cytostatic and antiestrogenic properties with human endometrial adenocarcinoma (ishikawa) cells." Cancer Res, 2001. 61(16): p. 6137-44.

[30] Chakraborty, S., A. S. Levenson, and P. K. Biswas, "Structural insights into resveratrol's antagonist and partial agonist actions on estrogen receptor alpha." BMC Struct Biol, 2013. 13: p. 27.

[31] Martins, M. J., M. R. Negrao, and C. Hipolito-Reis, "Alkaline phosphatase from rat liver and kidney is differentially modulated." Clin Biochem, 2001. 34(6): p. 463-8.

[32] Raisz, L. G., "The osteoporosis revolution marches on." J Orthop Sci, 2007. 12(4): p. 405-12.

[33] Bonnelye, E. and J. E. Aubin, "Differential expression of estrogen receptor-related receptor alpha and estrogen receptors alpha and beta in osteoblasts in vivo and in vitro." J Bone Miner Res, 2002. 17(8): p. 1392-400.

[34] Golub, E. E. and K. Boesze-Battaglia, "The role of alkaline phosphatase in mineralization." Current Opinion in Orthopaedics, 2007. 18(5): p. 444-448.

[35] Rao, L. G., et al., "Estrogen added intermittently, but not continuously, stimulates differentiation and bone formation in saos-2 cells." Biol Pharm Bull, 2003. 26(7): p. 936-45.

[36] Matsumoto, Y., et al., "Estrogen facilitates osteoblast differentiation by upregulating bone morphogenetic protein- 4 signaling." Steroids, 2013. 78(5): p. 513-20.

[37] Wei, A., et al., "Bmp-7 in combination with estrogen enhances bone formation in a fracture callus explant culture." Tohoku $\mathrm{J}$ Exp Med, 2010. 221(1): p. 61-8.

[38] Parikka, V., et al., "Estrogen responsiveness of bone formation in vitro and altered bone phenotype in aged estrogen receptoralpha-deficient male and female mice." Eur J Endocrinol, 2005. 152(2): p. 301-14.

[39] Monroe, D. G., et al., "Estrogen receptor isoform-specific regulation of endogenous gene expression in human osteoblastic cell lines expressing either eralpha or erbeta." J Cell Biochem, 2003. 90(2): p. 315-26.

[40] Guntur, A. R. and C. J. Rosen, "Bone as an endocrine organ." Endocr Pract, 2012. 18(5): p. 758-62.

[41] Guo, A. J., et al., "Kaempferol as a flavonoid induces osteoblastic differentiation via estrogen receptor signaling." Chin Med, 2012. 7: p. 10.

[42] Yen, M. L., et al., "Multilineage differentiation and characterization of the human fetal osteoblastic 1.19 cell line: A possible in vitro model of human mesenchymal progenitors." Stem Cells, 2007. 25(1): p. 125-31.

[43] Khalid, O., et al., "Modulation of runx2 activity by estrogen receptor-alpha: Implications for osteoporosis and breast cancer." Endocrinology, 2008. 149(12): p. 5984-95.

[44] Cao, Y., et al., "Osterix, a transcription factor for osteoblast differentiation, mediates antitumor activity in murine osteosarcoma." Cancer Res, 2005. 65(4): p. 1124-8.

[45] Zhu, F., et al., "The transcription factor osterix (sp7) regulates bmp6-induced human osteoblast differentiation." J Cell Physiol, 2012. 227(6): p. 2677-85.

[46] Kim, J. H., et al., "Aryl hydrocarbon receptor gene polymorphisms affect lung cancer risk." Lung Cancer, 2007. 56(1): p. 9-15.

[47] Wejheden, C., et al., "Osteopontin: A rapid and sensitive response to dioxin exposure in the osteoblastic cell line umr106." Biochem Biophys Res Commun, 2006. 341(1): p. 11620.

[48] Lee, L. L., et al., "Polycyclic aromatic hydrocarbons present in cigarette smoke cause bone loss in an ovariectomized rat model." Bone, 2002. 30(6): p. 917-23.

[49] Yun, C., et al., "Aryl hydrocarbon receptor antagonists mitigate the effects of dioxin on critical cellular functions in differentiating human osteoblast-like cells." Int J Mol Sci, 2018. 19(1)

[50] Singh, S. U., et al., "Inhibition of dioxin effects on bone formation in vitro by a newly described aryl hydrocarbon receptor antagonist, resveratrol." J Endocrinol, 2000. 167(1): p. 183-95.

[51] Ohtake, F., et al., "Modulation of oestrogen receptor signalling by association with the activated dioxin receptor." Nature, 2003. 423(6939): p. 545-50.

[52] Schlezinger, J. J., et al., "A role for the aryl hydrocarbon receptor in mammary gland tumorigenesis." Biol Chem, 2006. 387(9): p. 1175-87.

[53] Li, F. and C. Niyibizi, "Cells derived from murine induced pluripotent stem cells (ipsc) by treatment with members of tgfbeta family give rise to osteoblasts differentiation and form bone in vivo." BMC Cell Biol, 2012. 13: p. 35.

[54] Ehnert, S., et al., "Tgf-beta1 as possible link between loss of bone mineral density and chronic inflammation." PLoS One, 2010. 5(11): p. e14073.

[55] Sun, P., et al., "Bmp2/7 heterodimer is a stronger inducer of bone regeneration in peri-implant bone defects model than bmp2 or bmp7 homodimer." Dent Mater J, 2012. 31(2): p. 239-48.

[56] Zhou, S., et al., "Estrogens activate bone morphogenetic protein-2 gene transcription in mouse mesenchymal stem cells." Mol Endocrinol, 2003. 17(1): p. 56-66.

[57] Jang, W. G., et al., "Bmp2 protein regulates osteocalcin expression via runx2-mediated atf6 gene transcription." J Biol Chem, 2012. 287(2): p. 905-15. 
[58] Hyzy, S. L., et al., "Bmp2 induces osteoblast apoptosis in a maturation state and noggin-dependent manner." J Cell Biochem, 2012. 113(10): p. 3236-45.

[59] McBride, S. H., et al., "Long bone structure and strength depend on bmp2 from osteoblasts and osteocytes, but not vascular endothelial cells." PLoS One, 2014. 9(5): p. e96862.
[60] Phillips, F. M., et al., "In vivo bmp-7 (op-1) enhancement of osteoporotic vertebral bodies in an ovine model." Spine J, 2006. 6(5): p. 500-6. 